\title{
Randomized placebo-controlled trial comparing desloratadine and montelukast in monotherapy and desloratadine plus montelukast in combined therapy for chronic idiopathic urticaria
}

\author{
Gabriele Di Lorenzo, MD, ${ }^{\text {a }}$ Maria Luisa Pacor, MD, ${ }^{\mathrm{b}}$ Pasquale Mansueto, MD, \\ Maria Esposito Pellitteri, MD, ${ }^{a}$ Claudia Lo Bianco, MD, ${ }^{a}$ Vito Ditta, MD, ${ }^{\text {a }}$ \\ Nicola Martinelli, MD, ${ }^{\mathbf{b}}$ and Giovam Battista Rini, MD ${ }^{\mathbf{a}}$ Palermo and Verona, Italy
}

\begin{abstract}
Background: $\mathrm{H}_{1}$-receptor antagonists are considered to be particularly effective in reducing pruritus, and they are therefore recommended as first-line treatment in patients with chronic idiopathic urticaria (CIU). Recently, antileukotriene receptors have been used in patients with CIU, either administered as monotherapy or combined with $\mathrm{H}_{1}$-receptor antagonists.

Objective: We compared the clinical efficacy of $5 \mathrm{mg}$ of desloratadine administered once daily either as monotherapy or combined with a leukotriene antagonist, $10 \mathrm{mg}$ of montelukast daily, and $10 \mathrm{mg}$ of montelukast administered daily as monotherapy for the treatment of patients affected by CIU with placebo.

Methods: One hundred sixty patients aged 18 to 69 years (mean $\pm \mathrm{SD}, 43.9 \pm 13.4$ years) with a history of moderate CIU were selected. A randomized, double-blind, double-dummy, placebocontrolled, parallel-group study design was used. Patients were treated with $5 \mathrm{mg}$ of desloratadine once daily $(\mathrm{n}=40), 10 \mathrm{mg}$ of montelukast once daily $(\mathrm{n}=40), 5 \mathrm{mg}$ of desloratadine $(\mathrm{n}=40)$ in the morning plus montelukast in the evening, or matched placebo $(n=40)$. Assessment of treatment efficacy was based on scores of daily cutaneous symptoms evaluated reflectively and instantaneously.
\end{abstract}

Results: Only the group treated with desloratadine as monotherapy or as combined therapy concluded the whole study. Twenty-seven of the $\mathbf{4 0}$ patients in the montelukast group and 35 of the 40 patients in the placebo group discontinued the treatment. As reflective evaluation, all groups showed significant differences compared with the placebo group in terms of total symptom score, number of hives, and size of largest hive. In addition to the pruritus, only the groups treated with desloratadine as monotherapy or combined therapy showed significant differences compared with those receiving

\footnotetext{
From a Dipartimento di Medicina Clinica e delle Patologie Emergenti, Università di Palermo, and ${ }^{\mathrm{b}}$ Dipartimento di Medicina Clinica e Medicina Sperimentale, Università di Verona.

Supported by grants from MIUR (Ministero Italiano Università e Ricerca; fondi ex 60\%) to Gabriele Di Lorenzo and Maria Luisa Pacor, who received no support from the pharmaceutical industry.

Received for publication February 23, 2004; revised May 19, 2004; accepted for publication June 10, 2004

Reprint requests: Gabriele Di Lorenzo, MD, Dipartimento di Medicina Clinica e delle Patologie Emergenti, Via del Vespro 141, 90127 Palermo, Italy. E-mail: dilo601@unipa.it.

0091-6749/\$30.00

(C) 2004 American Academy of Allergy, Asthma and Immunology doi:10.1016/j.jaci.2004.06.018
}

placebo, whereas there were no differences between the montelukast and placebo groups. Finally, no differences were found between the desloratadine group and the desloratadine plus montelukast group. The instantaneous evaluation demonstrated similar results regarding the desloratadine group and the desloratadine plus montelukast group versus the placebo group, whereas there were no significant differences between the group treated with montelukast alone and the placebo group for pruritus and size of largest hive. No differences were found between the group treated with desloratadine alone and the desloratadine plus montelukast group.

Conclusions: The results of this comparative study demonstrate that desloratadine is highly effective for the treatment of patients affected by CIU. In addition, the regular combined therapy of desloratadine plus montelukast does not seem to offer a substantial advantage with respect to desloratadine as monotherapy in patients affected by moderate CIU. (J Allergy Clin Immunol 2004;114:619-25.)

Key words: Chronic idiopathic urticaria, desloratadine, montelukast

Urticarial episodes lasting longer than 6 weeks are considered chronic. ${ }^{1,2}$ In many patients the cause of chronic urticaria (CU) cannot be identified. In such cases urticaria is defined as chronic idiopathic urticaria (CIU). ${ }^{1,2}$ The histopathology of CIU and the cutaneous late-phase reaction to allergen are greatly similar. ${ }^{3}$ In patients with CIU, mast cells are activated, and local tissue inflammation (pruritus and swelling) is due to histamine release and possibly other mediators, such as leukotrienes. $\mathrm{H}_{1}$ receptor antagonists are the first-choice treatment for patients with $\mathrm{CIU},{ }^{1}$ but unfortunately, some patients do not benefit from these agents. ${ }^{1,2,4-8}$ Then the combination with a second agent is required, especially if $\mathrm{H}_{1}$-blockers are only partially effective.

In recent years, some reports have claimed a beneficial effect for leukotriene receptor antagonists (LT-RAs), such as montelukast and zafirlukast, as well as the 5-lipoxygenase inhibitor zileuton, for the treatment of patients with CU. ${ }^{9-25}$ The effects of LT-RAs in patients with CIU have been evaluated mostly in a heterogeneous population of patients. The majority of the studies reported in the literature are anecdotal reports, and only a few are 


\author{
Abbreviations used \\ ASA: Acetylsalicylic acid \\ CIU: Chronic idiopathic urticaria \\ CU: Chronic urticaria \\ LT-RA: Leukotriene receptor antagonist \\ NSAID: Nonsteroidal anti-inflammatory drug \\ TSS: Total symptom score
}

placebo-controlled studies. ${ }^{19-21,23,24}$ Among these studies, 4 demonstrated a beneficial effect of LT-RAs, ${ }^{19-21,24}$ whereas one demonstrated that LT-RAs do not provide a significant therapeutic benefit in patients with $\mathrm{CU}^{23}$ Patient selection is very important to investigate the efficacy of LT-RAs in CIU. Exclusion criteria are all the forms of CU secondary to any known cause or the forms of CU reactivated by drugs or food additives.

In this study we compared the clinical efficacy of desloratadine, a new $\mathrm{H}_{1}$-receptor antagonist, administered once daily as monotherapy or combined with a leukotriene antagonist (montelukast). Determining the efficacy of montelukast as monotherapy for the treatment of patients affected by CIU was also an objective of the present study.

\section{METHODS}

\section{Patients}

We selected 160 adult patients (49 male and 111 female patients; age range, 18-69 years) with CIU from our outpatient clinic at the University Hospitals in Palermo and Verona. CIU was defined as the presence of urticarial lesions for more than 6 weeks' duration in patients with more than 3 episodes of urticaria a week without any secondary known causes. The presence of urticarial skin lesions was confirmed clinically. In all patients we have excluded the presence of positive skin test results to autologous serum and the appearance of urticaria after the administration of acetylsalicylic acid (ASA) or nonsteroidal anti-inflammatory drugs (NSAIDs) or after a challenge with food additives. Patients affected by physical or allergic urticaria or by urticaria-vasculitis were also excluded from the study. The other exclusion criteria of the study were the following: pregnancy, breast-feeding, important systemic or psychiatric disease, and habitual use of corticosteroids or LT-RAs for 2 months before entry into the study or use of oral corticosteroids in the month before the beginning of the study. Before the study began, approval was obtained from the ethics committees of the 2 centers involved. Written informed consent was obtained from all enrolled patients.

\section{Study design}

A randomized, double-blind, double-dummy, placebo-controlled, parallel-group study design was used. In each center patients received the following treatments: (1) $5 \mathrm{mg}$ of desloratadine daily in the morning plus placebo of montelukast in the evening; (2) $5 \mathrm{mg}$ of desloratadine daily in the morning plus $10 \mathrm{mg}$ of montelukast in the evening; (3) placebo of desloratadine in the morning plus $10 \mathrm{mg}$ of montelukast 1 day in the evening; or (4) placebo of desloratadine in the morning plus placebo of montelukast in the evening.

The pharmacist of the University Hospital of Verona prepared a specific set with the treatments to be used for the study. The investigators and patients were blinded with respect to the contents of each set. The pharmacist used commercially available tablets of desloratadine (Aerius; Schering-Plough, Italy), montelukast (Singulair; Merck Sharp and Dohme, Italy), or placebo. All treatments were administered by a person unaware of who was participating in the study. Rescue medication included loratadine tablets (Clarytin, $10 \mathrm{mg}$; Shering-Plough, Italy). No other medication for urticaria was permitted during the trial.

The treatment period started after the clinical diagnosis of CIU (see below), without any run-in period. Patients were treated for 6 weeks.

Each patient attended the clinic on 4 different occasions after the diagnostic procedure. These included an initial clinic visit (visit 1), a second visit after 3 weeks of treatment (visit 2), a final visit after 6 weeks of treatment (visit 3), and a visit 2 weeks after the end of the treatment period (follow-up, visit 4).

At visit 1 , symptom scores of urticaria were assessed by patients by means of a visual analog scale (0-9). Enrolled patients received a daily record diary for cutaneous symptoms. The study was conducted during 2002. All groups for each treatment included 40 patients: 20 patients (for each treatment) enrolled in Palermo and 20 patients (for each treatment) enrolled in Verona.

Before the beginning of the treatment period, a clinical history was recorded for each patient, and physical examinations and standard and specific laboratory investigations for urticaria were also performed. In particular, the following tests were performed: skin prick test for common aeroallergens and food allergens ${ }^{26}$; hematologic parameter assessment (hemoglobin, red blood cell, platelet, and white blood cell counts); biochemical assessment (serum electrolytes [sodium and potassium]); indices of renal function (creatinine, urea, and urine analysis) and hepatic function (alkaline phosphatase, aspartate aminotransferase, alanine aminotransferase, and $\gamma$-glutamil-transpeptidase); glucose-fasting testing; and C-reactive protein, serum total IgE, and antinuclear and antithyroid antibody measurement. Finally, in all patients we performed an intradermal test with auto$\operatorname{logous~serum~}^{2}$ and double-blind placebo-controlled challenges with ASA, NSAIDs, and food additives, as previously described. ${ }^{19,26,27}$

\section{Efficacy assessments}

Throughout the study, disease activity was assessed by the patients with a scoring system for CIU on the basis of specific signs and symptoms. ${ }^{28}$ Patients recorded the scores on their diary cards. Instruction on how to perform the assessment was provided at the time of screening. CIU signs and symptom scores were evaluated by using 4-point scales (0-3) for pruritus, number of hives, size of largest hive, interference with sleep, and interference with daily activities (Table I). ${ }^{28}$ Twice daily (morning and evening) patients scored pruritus, number of hives, and size of largest hive over the preceding 12 hours (reflective) and immediately at the time of assessment (instantaneous). These assessments were performed on awakening (before dosing) and 12 hours later. Reflective assessments of interference with sleep and daily activities were scored in the morning and in the evening only, respectively.

\section{Safety assessments}

Vital signs were recorded at all visits, whereas electrocardiography and laboratory tests were performed at screening and visit 3 . All adverse events were recorded and graded for severity and potential relation to the medications used in the study.

Safety evaluations included the incidence of treatment-induced or emergency adverse events, discontinuations because of adverse events, and changes from baseline in vital signs, laboratory parameters, and electrocardiographic intervals.

\section{Statistical analysis}

The primary assessment of efficacy was based on the differences between each treatment group versus placebo for total symptom score 
TABLE I. Individual signs and symptom score system ${ }^{28}$

\begin{tabular}{|c|c|c|c|c|c|}
\hline Score & Pruritus & No. of hives & Size of largest hive (cm) & $\begin{array}{l}\text { Interference with } \\
\text { sleep }\end{array}$ & $\begin{array}{l}\text { Interference with } \\
\text { daily activities }\end{array}$ \\
\hline 0 & None & None & None & None & None \\
\hline 1 & $\begin{array}{l}\text { Mild, minimal } \\
\text { awareness, easily tolerated }\end{array}$ & $1-6$ & $<1.25$ & $\begin{array}{l}\text { Mild, not troublesome, } \\
\text { adequate sleep }\end{array}$ & $\begin{array}{l}\text { Mild, not troublesome, } \\
\text { little effect on activity }\end{array}$ \\
\hline 2 & $\begin{array}{l}\text { Moderate, definite awareness, } \\
\text { bothersome but tolerable }\end{array}$ & $7-12$ & $1.25-2.5$ & $\begin{array}{l}\text { Moderate, awoke } \\
\text { occasionally, average sleep }\end{array}$ & $\begin{array}{l}\text { Moderate, some interference } \\
\text { with activity }\end{array}$ \\
\hline 3 & Severe, difficult to tolerate & $>12$ & $>2.5$ & $\begin{array}{l}\text { Severe, substantial interference } \\
\text { with sleep, poor sleep }\end{array}$ & $\begin{array}{l}\text { Severe, daily activities } \\
\text { substantially or completely } \\
\text { curtailed }\end{array}$ \\
\hline
\end{tabular}

TABLE II. Characteristics of patients

\begin{tabular}{lccccc}
\hline & Desloratadine & Montelukast & Desloratadine plus montelukast & Placebo & $\boldsymbol{P}$ value \\
\hline No. & 40 & 40 & 40 & 40 & \\
Age (y), mean (minimum and maximum) & $44.0(18-63)$ & $44.4(18-64)$ & $43.7(20-69)$ & $43.6(20-65)$ & NS \\
Sex (M/F) & $13 / 27$ & $12 / 28$ & $13 / 27$ & $11 / 29$ & NS \\
Onset of urticaria (mo) & $16.6(6-43)$ & $16.3(7-34)$ & $16.9(10-42)$ & $17.0(7-44)$ & NS \\
Symptoms,mean (minimum and maximum) & $6.4(5-9)$ & $6.9(4-9)$ & $6.3(5-9)$ & $7.1(4-9)$ & NS \\
\hline
\end{tabular}

NS, Not significant.

(TSS) and for every symptom of the urticaria. The secondary assessment was based on the differences between the group treated with desloratadine and the group treated with desloratadine plus montelukast. Data are presented as means and 95\% CIs of the means of individual score. Adjusted values were subsequently averaged by patient over the entire observation period. Averages by patient were examined by using a mixed-effects ANOVA model, with the treatments (as fixed effect) and the centers (as random effect) as the main values. F values were calculated by using the mean squares of the interaction "centers $\times$ treatments" as the error term. Differences between means were performed by using the Bonferroni multiple range test (set at 95\% CI). Power analysis on post hoc comparisons was performed with the GPower software package. ${ }^{29}$ Comparisons are only denoted as being significant $(P<.05,2$ tail $)$ or not significant if our sample size exceeded the minimum sample size resulting from a power analysis at a $\beta$ value of .80

\section{RESULTS}

One hundred sixty patients were randomized to treatments, with 40 patients for each treatment (see above). The baseline characteristics with respect to duration of urticaria and age of patients are reported in Table II. Only the group treated with desloratadine as monotherapy or combined therapy concluded the whole study. Twenty-seven of forty patients in the montelukast group and 35 of 40 patients in the placebo group discontinued the treatment.

\section{Assessment of efficacy}

Fig 1 and Table III show TSS, pruritus, number of hives, and size of largest hive in reflective and instantaneous evaluations. Fig 2 and Table IV show interference with daily activities and interference with sleep, respectively. Data are reported as means and $95 \%$ CIs of the means of individual scores during the treatment period and as the mean difference (95\%CI for difference) between the treatments.

Reflective evaluation. All groups showed significant differences compared with placebo in terms of TSS, number of hives, and size of largest hive. In addition to the pruritus, only the groups treated with desloratadine as monotherapy or combined therapy showed significant differences compared with those receiving placebo, but there were no differences between the groups treated with montelukast and placebo.

Comparing the group treated with desloratadine alone and the group treated with montelukast alone, we found significant differences for TSS, pruritus, number of hives, and size of largest hive $(P<.001, P<.001, P=.017$, and $P=.003$, respectively). The comparisons between the groups treated with desloratadine plus montelukast and the group treated with montelukast alone showed significant difference for TSS, pruritus, number of hives, and size of largest hive $(P<.001, P<.001, P=.01$, and $P=.003$, respectively).

No differences were found between the group treated with desloratadine alone and the group treated with desloratadine plus montelukast.

Instantaneous evaluation. The results of the analysis of the instantaneous evaluation regarding TSS, pruritus, number of hives, and size of largest hive are similar to the results of reflective evaluation. Examining the group treated with montelukast alone versus the group treated with placebo, we found no difference for pruritus and size of largest hive, whereas there were significant differences for TSS $(P=.005)$ and number of hives $(P=.001)$.

In the group treated with desloratadine alone, we found significant differences for TSS $(P<.001)$, pruritus $(P=.003)$, number of hives $(P=.002)$, and size of largest 
TABLE III. Values for desloratadine, montelukast, desloratadine plus montelukast, and placebo and mean differences between the treatments for TSS (out of 9) pruritus (out of 3 ), number of hives (out of 3), size of hives (out of 3 )

\begin{tabular}{|c|c|c|c|c|}
\hline Treatments & TSS & Pruritus & Number of hives & Size of hives \\
\hline & \multicolumn{4}{|c|}{ Subjective symptoms of urticaria (reflective evaluation), mean $(95 \% \mathrm{CI})$} \\
\hline DSL & $1.49(1.44-1.53)$ & $0.50(0.48-0.51)$ & $0.40(0.38-0.43)$ & $0.41(0.3$ \\
\hline MSK & $2.65(2.54-2$ & ) & & \\
\hline DSL plus MSK & $1.50(1.44-1.57)$ & $0.51(0.49-0.53)$ & $0.40(0.38-0.44)$ & $0.42(0.40-0.44)$ \\
\hline \multirow[t]{2}{*}{ PLA } & $3.26(3.17-3.36)$ & $1.19(1.15-1.22)$ & $1.02(0.97-1.07)$ & $0.88(0.84-0.93)$ \\
\hline & \multicolumn{4}{|c|}{ Comparisons: mean differences between the treatments (95\% CI for difference); reflective evaluation } \\
\hline DSL vs PLA & $\begin{aligned}-1.77(-1.89 \text { to }-1.66) & \\
& P<.001\end{aligned}$ & $\begin{array}{l}-0.68(-0.72 \text { to }-0.65) \\
P<.001\end{array}$ & $\begin{array}{l}-0.61(-0.67 \text { to }-0.56) \\
P=.001\end{array}$ & $\begin{array}{c}-0.46(-0.52 \text { to }-0.41) \\
P=.001\end{array}$ \\
\hline MSK vs PLA & $\begin{aligned}-0.61 & (-0.72 \text { to }-0.49) \\
& P<.001\end{aligned}$ & $\begin{array}{l}0.01(-0.02 \text { to } 0.05) \\
P=\mathrm{NS}\end{array}$ & $\begin{array}{l}-0.40(-0.45 \text { to }-0.34) \\
\quad P=.001\end{array}$ & $\begin{array}{c}-0.22(-0.28 \text { to }-0.17) \\
P=.003\end{array}$ \\
\hline DSL plus MSK vs PLA & $\begin{array}{c}-1.76(-1.87 \text { to } 1.64) \\
P<.001\end{array}$ & $\begin{aligned}-0.67(-0.71 \text { to }-0.63) \\
P<.001\end{aligned}$ & $\begin{array}{l}-0.61(-0.67 \text { to }-0.56) \\
P=.001\end{array}$ & $\begin{aligned}-0.46 & (-0.51 \text { to }-0.42) \\
& P<.001\end{aligned}$ \\
\hline DSL vs MSK & $\begin{aligned}-1.66(-1.28 \text { to }-1.05) & \\
& P<.001\end{aligned}$ & $\begin{array}{l}-0.70(-0.74 \text { to }-0.66) \\
P<.001\end{array}$ & $\begin{array}{l}-0.21(-0.27 \text { to }-0.16) \\
P=.017\end{array}$ & $\begin{array}{l}-0.24(-0.29 \text { to }-0.19), \\
P=.003\end{array}$ \\
\hline MSK vs DSL plus MSK & $\begin{array}{c}1.14 \text { (1.03 to } 1.26) \\
\quad P<.001\end{array}$ & $\begin{array}{c}0.69(0.65 \text { to } 0.63) \\
P<.001\end{array}$ & $\begin{array}{c}0.22 \text { (0.16 to } 0.30) \\
\quad P=.01\end{array}$ & $\begin{array}{l}0.24 \text { ( } 0.18 \text { to } 0.29) \\
\quad P=.003\end{array}$ \\
\hline \multirow[t]{2}{*}{ DSL vs DSL plus MSK } & $\begin{array}{l}-0.01(-0.13 \text { to } 0.09) \\
P=\text { NS }\end{array}$ & $\begin{array}{l}-0.01(-0.05 \text { to }-0.02) \\
P=\text { NS }\end{array}$ & $\begin{array}{l}-0.0007(-0.05 \text { to } 0.05) \\
\quad P=\mathrm{NS}\end{array}$ & $\begin{array}{l}-0.0001(-0.05 \text { to } 0.05), \\
\quad P=\text { NS }\end{array}$ \\
\hline & \multicolumn{4}{|c|}{ Subjective symptoms of urticaria (instantaneous evaluation), mean $(95 \% \mathrm{CI})$} \\
\hline DSL & $1.81(1.77-1.85)$ & $0.45(0.43-0.48)$ & $0.54(0.52-0.56)$ & $0.67(0$ \\
\hline MSK & 2.86( & & & 0.93 \\
\hline DSL $\mathrm{p}$ & 1.75( & & & 0.6 \\
\hline \multirow[t]{2}{*}{ PLA } & $3.41(3.32-3.49)$ & $1.17(1.11-1.23)$ & $1.14(1.11-1.84)$ & $0.95(0.90-1.01)$ \\
\hline & \multicolumn{4}{|c|}{ Comparisons: mean differences between the treatments (95\% CI for difference); instantaneous evaluation } \\
\hline DSL vs PLA & $\begin{aligned}-1.59(-1.68 \text { to }-1.50) & \\
& P<.001\end{aligned}$ & $\begin{array}{l}-0.71(-0.76 \text { to }-0.66) \\
P=.002\end{array}$ & $\begin{aligned}-0.60 & (-0.64 \text { to }-0.56) \\
& P<.001\end{aligned}$ & $\begin{array}{c}-0.27(-0.32 \text { to }-0.22), \\
P=.008\end{array}$ \\
\hline MSK vs PLA & $\begin{array}{l}-0.54(-0.63 \text { to }-0.45) \\
\quad P=.005\end{array}$ & $\begin{array}{l}-0.14(-0.19 \text { to } 0.01) \\
P=\text { NS }\end{array}$ & $\begin{array}{l}-0.37(-0.41 \text { to }-0.32) \\
P=.001\end{array}$ & $\begin{array}{l}-0.02(-0.07 \text { to } 0.02) \\
P=\text { NS }\end{array}$ \\
\hline DSL plus MSK vs PLA & $\begin{aligned}-1.65(-1.74 \text { to } 1.56) & \\
& P<.001\end{aligned}$ & $\begin{array}{l}-0.64(-0.69 \text { to }-0.59) \\
P=.002\end{array}$ & $\begin{aligned}-0.64(-0.68 \text { to }-0.60) \\
P<.001\end{aligned}$ & $\begin{aligned}-0.32(-0.36 \text { to }-0.27) \\
P<.001\end{aligned}$ \\
\hline DSL vs MSK & $\begin{array}{l}-1.04(-1.14 \text { to }-0.95) \\
\quad P=.001\end{array}$ & $\begin{array}{l}-0.57(-0.62 \text { to }-0.51) \\
P=.003\end{array}$ & $\begin{array}{l}-0.23(-0.27 \text { to }-0.18) \\
P=.002\end{array}$ & $\begin{array}{l}-0.25(-0.29 \text { to }-0.20), \\
P=.008\end{array}$ \\
\hline MSK vs DSL plus MSK & $\begin{array}{c}1.10(1.01 \text { to } 1.20), \\
P=.001\end{array}$ & $\begin{array}{c}0.50(0.45 \text { to } 0.55) \\
P=.004\end{array}$ & $\begin{array}{c}0.27(0.22 \text { to } 0.31) \\
\quad P=.001\end{array}$ & $\begin{array}{c}0.29(0.24 \text { to } 0.34) \\
P=.007\end{array}$ \\
\hline DSL vs DSL plus MSK & $\begin{array}{l}0.05 \text { ( }-0.03 \text { to } 0.15), \\
\quad P=\mathrm{NS}\end{array}$ & $\begin{array}{l}-0.006(-0.11 \text { to } 0.01) \\
\quad P=\text { NS }\end{array}$ & $\begin{array}{c}0.004 \text { ( }-0.03 \text { to } 0.08) \\
P=\text { NS }\end{array}$ & $\begin{array}{l}0.04(-0.004 \text { to } 0.09), \\
\quad P=\text { NS }\end{array}$ \\
\hline
\end{tabular}

$D S L$, Desloratadine; $M S K$, montelukast; PLA, placebo; NS, not significant.

TABLE IV. Mean values for desloratadine, montelukast, desloratadine plus montelukast, and placebo and mean differences between the treatments for interference with sleep (out of 3 ) and interference with daily activities (out of 3)

\begin{tabular}{lcc}
\hline Treatments & Interference with sleep $\mathbf{9 5 \%} \mathbf{~ C l )}$ & Interference with daily activities $\mathbf{( 9 5 \%} \mathbf{~} \mathbf{~})$ \\
\hline DSL & $0.60(0.58-0.62)$ & $0.35(0.33-0.37)$ \\
MSK & $1.00(0.97-1.03)$ & $0.41(0.37-0.44)$ \\
DSL plus MSK & $9.72(0.69-0.75)$ & $0.40(0.38-0.42)$ \\
PLA & $1.03(0.99-1.07)$ & $0.87(0.84-0.90)$ \\
& Comparisons: mean differences between the treatments $(95 \%$ CI for difference) \\
DSL vs PLA & $-0.43(-0.47$ to -0.39$), P=.003$ & $-0.52(-0.55$ to -0.48$), P=.001$ \\
MSK vs PLA & $-0.03(-0.07$ to 0.01$), P=\mathrm{NS}$ & $-0.46(-0.50$ to -0.43$), P=.002$ \\
DSL plus MSK vs PLA & $-0.31(-0.35$ to -0.27$), P=.007$ & $-0.47(-0.50$ to -0.44$), P=.002$ \\
DSL vs MSK & $-0.40(-0.44$ to -0.35$), P=.003$ & $-0.06(-0.09$ to -0.02$), P=\mathrm{NS}$ \\
MSK vs DSL plus MSK & $0.28(0.24$ to 0.32$), P=.01$ & $0.0002(-0.03$ to 0.03$), P=\mathrm{NS}$ \\
DSL vs DSL plus MSK & $-0.11(-0.16$ to -0.07$), P=\mathrm{NS}$ & $-0.05(-0.08$ to -0.01$), P=\mathrm{NS}$
\end{tabular}

DSL, Desloratadine; MSK, montelukast; PLA, placebo; NS, Not significant. 
(a)

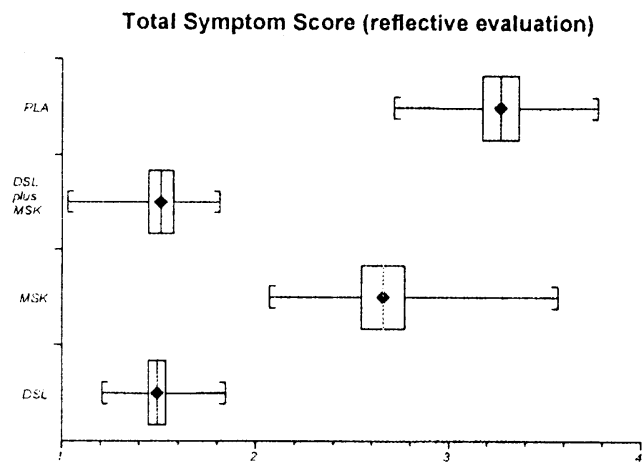

(b)

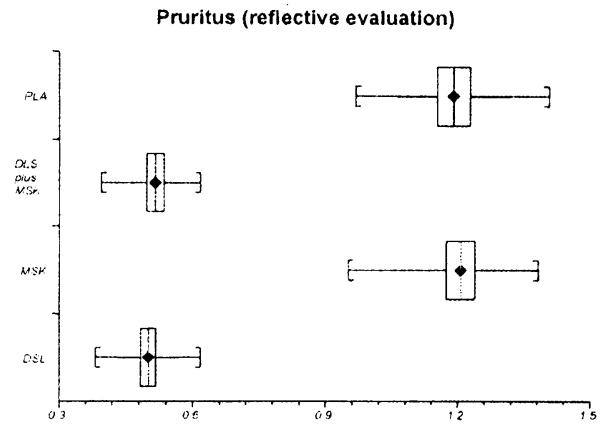

(c)

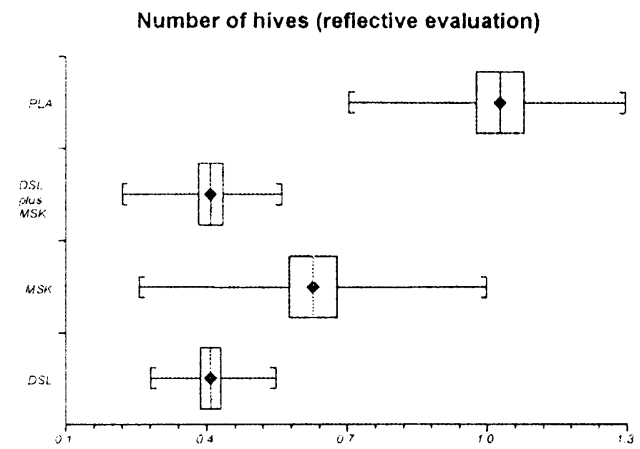

(d)

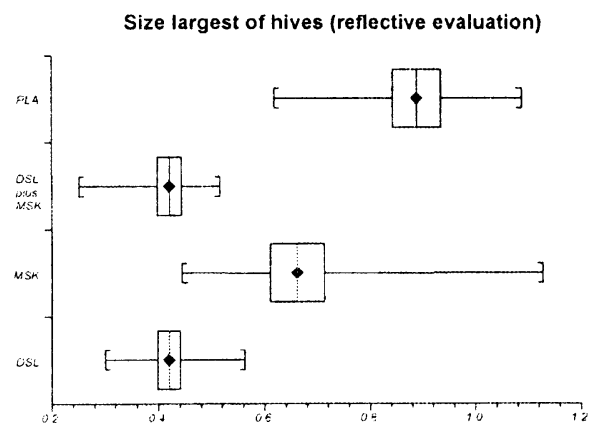

(e)



(c)

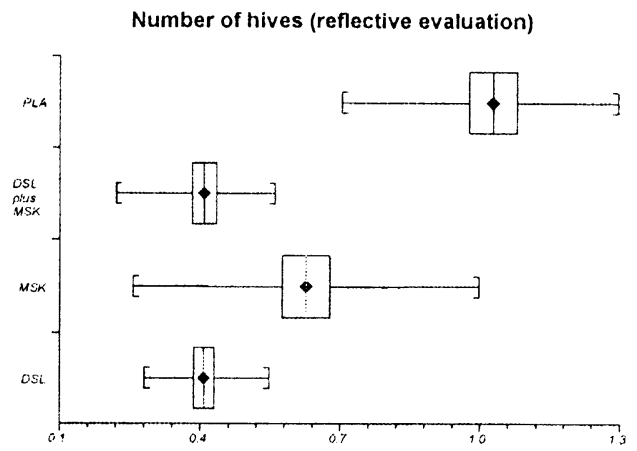

(g)

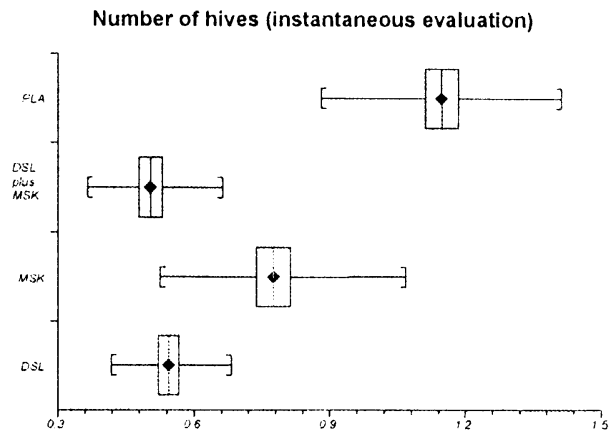

(h)

Size largest of hives (instantaneous evaluation)

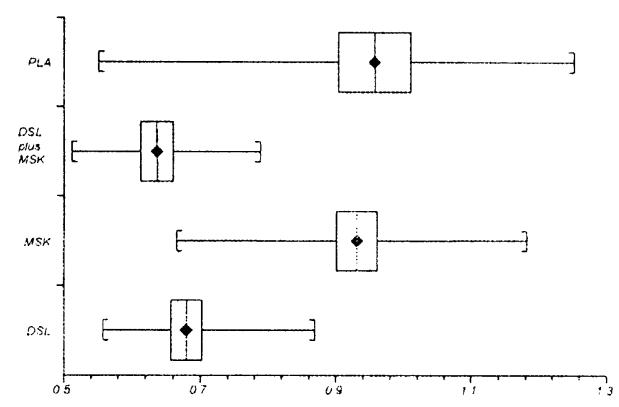

FIG 1. Subjective symptoms of urticaria, TSS, pruritus, numbers of hives, and size of largest hive as reflective and instantaneous evaluations. Data are reported as means with $95 \% \mathrm{Cls}$ of the mean of individual scores during the treatment period. The comparison between treatments is reported in Table III. 
(a)



(b)

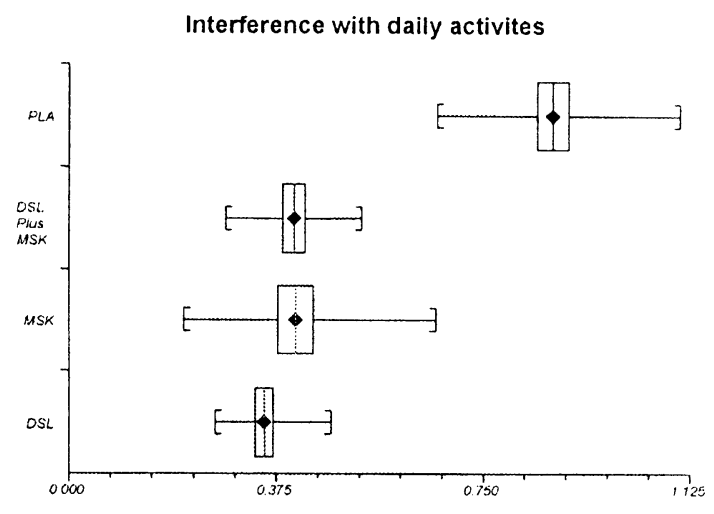

FIG 2. Interference with sleep and interference with daily activities. Data are reported as means with $95 \% \mathrm{Cls}$ of the mean of the individual scores during the treatment period. The comparison between treatments is reported in Table IV.

hive $(P=.008)$ in comparison with the group treated with montelukast alone. We obtained similar results comparing the group treated with desloratadine plus montelukast and the group treated with montelukast alone for TSS $(P=.001)$, pruritus $(P=.004)$, number of hives $(P=.001)$, and size of largest hive $(P=.007)$. We found no significant differences between the group treated with desloratadine alone and the group treated with desloratadine plus montelukast.

Interference with sleep. Regarding interference with sleep, significant differences were found between the groups treated with desloratadine, both as monotherapy and as combined therapy with montelukast, in comparison with the placebo group $(P=.003$ and $P=.007$, respectively). Comparing the group treated with desloratadine alone versus the group treated with montelukast alone, we found significant differences $(P=.003)$. Also, significant differences were found comparing those treated with montelukast alone versus those treated with desloratadine plus montelukast $(P=.01)$. In addition, no differences were found between the group treated with montelukast alone versus the group treated with placebo and between the group treated with desloratadine alone versus the group treated with desloratadine plus montelukast.

Interference with daily activities. Considering daily activities, we found significant differences between all active treatments and the placebo group. We found no differences among the groups treated with desloratadine alone, montelukast alone, and desloratadine plus montelukast.

Use of rescue medication. The use of rescue medication, expressed as the median number of days without the use of loratadine tablets, was significantly lower in the groups treated with desloratadine as monotherapy (90.6 days) or combined therapy (91.0 days) than in the montelukast-treated group $(45.2$ days, $P<.001)$ or the placebo-treated group (54.0 days, $P<.001)$. We found no differences between the group treated with desloratadine and the group treated with desloratadine plus montelukast and between the group treated with montelukast and the group treated with placebo.

\section{Safety}

A low incidence of adverse events was observed in the study. All adverse events were rated as mild. Exacerbation of urticarial symptoms was reported in 27 patients in the group treated with montelukast and in 35 patients in the group treated with placebo.

\section{DISCUSSION}

The results of this study demonstrate that desloratadine administered once daily is more effective than montelukast for the treatment of urticarial symptoms in patients with moderate CIU. The combination of desloratadine with montelukast failed to produce a substantial advantage for urticarial symptoms in comparison with desloratadine administered in monotherapy. Moreover, treatment with montelukast as monotherapy failed to control the urticarial symptoms, such as pruritus, in our study in patients affected by CIU.

In this study we evaluated only patients affected by moderate CIU. We excluded patients with positive challenge results to ASA, NSAIDs, or food additive; those with positive cutaneous test results to autologous serum; or both. We also excluded patients who reported an aggravation of their symptoms through pressure. Therefore the absence of these triggers indicates the presence of an idiopathic form of urticaria. Angioedema was rarely present in this group of patients. This is an important difference compared with some of the previous reports, in which patients were selected without precise characteristics. $^{20,21,23}$

In patients with moderate CIU, the role of leukotrienes is probably rather insignificant. ${ }^{24}$ In a previous study we examined patients with CIU and with CU who had ASA or food additive sensitivity, determining urinary metabolite concentrations of both histamine and leukotrienes. ${ }^{30}$ The 2 
groups of patients affected by CU had markedly increased urinary concentrations of methylhistamine at baseline, thus allowing a clear distinction from the control group (healthy volunteers). On the contrary, when we compared urinary leukotriene $\mathrm{E}_{4}$ levels in the same subjects, we found no difference at baseline in the 3 groups but an increase in leukotriene levels after challenge only in patients with ASA or food additive sensitivity. These results might also explain the low clinical response to therapy with LT-RAs in patients with CIU with no welldefined triggers. Recently, the results of the study by Bagenstose et $\mathrm{al}^{24}$ would confirm that the treatment of combined therapy with $\mathrm{H}_{1}$-receptor antagonists and LTRAs is effective only in patients with autoimmune and more severe urticaria (positive skin test response to autologous serum). ${ }^{24,31}$

Finally, we should consider the economic aspect of the treatments: the cost of an anti- $\mathrm{H}_{1}$-receptor treatment is $0.53 €$ per day, whereas the daily cost of an antileukotriene receptor treatment is $2.02 €$ per day. Particularly, the consumption of rescue medication (loratadine) is similar in patients treated with montelukast as monotherapy and in patients treated with placebo, and these data confirm that histamine is the most important mediator of the CIU.

In conclusion, the results of this comparative study demonstrate that desloratadine, regularly administered once daily, is effective for the treatment of urticarial symptoms, confirming the results of other studies. ${ }^{28,32}$

Statistical advice was kindly provided by Full Professor Antonio Motisi (Dipartimento di Coltivazioni Arboree, Università di Palermo).

\section{REFERENCES}

1. Greaves M. Chronic urticaria. J Allergy Clin Immunol 2000;105:664-72.

2. Kaplan AP. Clinical practice. Chronic urticaria and angioedema. N Engl J Med 2002;346:175-9.

3. Grattan CE, Boon AP, Eady RA, Winkelmann RK. The pathology of the autologous serum skin test response in chronic urticaria resembles IgE-mediated late-phase reactions. Int Arch Allergy Appl Immunol 1990;93:198-204.

4. Goldsobel AB, Rohr AS, Siegel SC, Spector SL, Katz RM, Rachelefsky GS, et al. Efficacy of doxepin in the treatment of chronic idiopathic urticaria. J Allergy Clin Immunol 1986;78:867-73.

5. Fradin MS, Ellis CN, Goldfarb MT, Voorhees JJ. Oral cyclosporine for severe chronic idiopathic urticaria and angioedema. J Am Acad Dermatol 1991;25:1065-7.

6. Grattan CE, O'Donnell BF, Francis DM, Niimi N, Barlow RJ, Seed PT, et al. Randomized double-blind study of cyclosporin in chronic 'idiopathic' urticaria. Br J Dermatol 2000;143:365-72.

7. O’Donnell BF, Barr RM, Black AK, et al. Intravenous immunoglobulin in autoimmune chronic urticaria. Br J Dermatol 1998;138:101-6.

8. Gaig P, Garcia-Ortega P, Enrique E, Richart C. Successful treatment of chronic idiopathic urticaria associated with thyroid autoimmunity. J Investig Allergy Clin Immunol 2000;10:342-5.

9. Spector S, Tan RA. Antileukotrienes in chronic urticaria. J Allergy Clin Immunol 1998;101:572.

10. Ellis MH. Successful treatment of chronic urticaria with leukotriene antagonists. J Allergy Clin Immunol 1998;102:876-7.

11. Norris JG, Sullivan TJ. Leukotrienes and cytokines in steroid dependent chronic urticaria. J Allergy Clin Immunol 1998;1(suppl):S128.
12. Chu TJ, Warren MS. Zafirlukast ACCOLATE in the treatment of chronic idiopathic urticaria-a case series. J Allergy Clin Immunol 1998; 1(suppl):S155.

13. Bensch GW, Borish L. Leukotriene receptor antagonists in the treatment of chronic idiopathic urticaria. J Allergy Clin Immunol 1999;1(suppl): S154.

14. Berkun Y, Shalit M. Successful treatment of delayed pressure urticaria with montelukast. Allergy 2000;55:203-4

15. Asero R. Leukotriene receptor antagonists may prevent NSAID-induced exacerbations in patients with chronic urticaria. Ann Allergy Asthma Immunol 2000;85:156-7.

16. Tedeschi A, Suli C, Lorini M, Airaghi L. Successful treatment of chronic urticaria. Allergy 2000;55:1097-8.

17. Hani N, Hartmann K, Casper C, Peters T, Schneider LA, Hunzelmann N, et al. Improvement of cold urticaria by treatment with the leukotriene receptor antagonist montelukast. Acta Derm Venereol 2000; $80: 229$

18. Asero R, Tedeschi A, Lorini M. Leukotriene receptor antagonists in chronic urticaria. Allergy 2001;56:456-7.

19. Pacor ML, Di Lorenzo G, Corrocher R. Efficacy of leukotriene receptor antagonist in chronic urticaria. A double-blind, placebo-controlled comparison of treatment with montelukast and cetirizine in patients with chronic urticaria with intolerance to food additive and/or acetylsalicylic acid. Clin Exp Allergy 2001;31:1607-14.

20. Nettis E, Dambra P, D’Oronzio L, Loria MP, Ferrannini A, Tursi A. Comparison of montelukast and fexofenadine for chronic idiopathic urticaria. Arch Dermatol 2001;137:99-100.

21. Erbagci Z. The leukotriene receptor antagonist montelukast in the treatment of chronic idiopathic urticaria: a single-blind, placebo-controlled, crossover clinical study. J Allergy Clin Immunol 2002;110: 484-8.

22. Ohnishi-Inoue Y, Mitsuya K, Horio T. Aspirin-sensitive urticaria: provocation with a leukotriene receptor antagonist. $\mathrm{Br} \mathrm{J}$ Dermatol 1998; 138:483-5.

23. Reimers A, Pichler C, Helbling A, Pichler WJ, Yawalkar N. Zafirlukast has no beneficial effects in the treatment of chronic urticaria. Clin Exp Allergy 2002;32:1763-8

24. Bagenstose SE, Levin L, Bernstein JA. The addition of zafirlukast to cetirizine improves the treatment of chronic urticaria in patients with positive autologous serum skin test results. J Allergy Clin Immunol 2004; 113:134-40.

25. Perez C, Sanchez-Borges M, Capriles E. Pretreatment with montelukast blocks NSAID-induced urticaria and angioedema. J Allergy Clin Immunol 2001;108:1060-1.

26. Pacor ML, Di Lorenzo G, Martinelli N, Mansueto P, Rini GB, Corrocher R. Monosodium benzoate hypersensitivity in subjects with perennial rhinitis. Allergy 2004;59:192-7.

27. Pacor ML, Di Lorenzo G, Biasi D, Barbagallo M, Corrocher R. Safety of rofecoxib in subjects with a history of adverse cutaneous reactions to aspirin and/or non-steroidal anti-inflammatory drugs. Clin Exp Allergy 2002;32:397-400.

28. Monroe E, Finn A, Patel P, Guerrero R, Ratner P, Bernstein D. Desloratadine Urticaria Study Group. Efficacy and safety of desloratadine $5 \mathrm{mg}$ once daily in the treatment of chronic idiopathic urticaria: a double-blind, randomized, placebo-controlled trial. J Am Acad Dermatol 2003;48:535-41.

29. Erdfelder E, Faul F, Buchner A. GPower: a general power analysis program. Behav Res Methods Instrum Comput 1996;28:1-11.

30. Di Lorenzo G, Pacor ML, Vignola AM, Profita M, Esposito-Pellitteri M, Biasi D, et al. Urinary metabolites of histamine and leukotrienes before and after placebo-controlled challenge with ASA and food additives in chronic urticaria patients. Allergy 2002;57:1180-6.

31. Sabroe RA, Seed PT, Francis DM, Barr RM, Black AK, Greaves MW. Chronic idiopathic urticaria: comparison of the clinical features of patients with and without anti-FcepsilonRI or anti-IgE autoantibodies. J Am Acad Dermatol 1999;40:443-50.

32. Ring J, Hein R, Gauger A, Bronsky E, Miller B. Once-daily desloratadine improves the signs and symptoms of chronic idiopathic urticaria: a randomized, double-blind, placebo-controlled study. Int J Dermatol 2001;40:72-6 